Proceedings of the International Symposium on Physics of Materials (ISPMA 14), September 10-15, 2017, Prague

\title{
Anisotropic Elasticity of Ceramic Micro-Scaffolds Fabricated by Robocasting
}

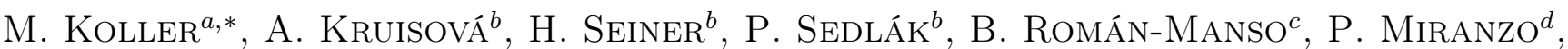 \\ M. BELMONTE ${ }^{d}$ AND M. LANDA ${ }^{b}$ \\ ${ }^{a}$ Faculty of Nuclear Sciences and Physical Engineering, Czech Technical University in Prague, \\ Trojanova 13, 12000 Praha 2, Czech Republic \\ ${ }^{b}$ Institute of Thermomechanics, Czech Academy of Sciences, Dolejškova 1402/5, 18200 Praha 8, Czech Republic \\ ${ }^{c}$ School of Engineering and Applied Sciences, Harvard University, 52 Oxford Str., 02318 Cambridge MA, USA \\ ${ }^{d}$ Institute of Ceramics and Glass (ICV-CSIC), Kelsen 5, 28049 Madrid, Spain
}

\begin{abstract}
Anisotropic elastic and acoustic properties of robocast ceramic scaffolds are calculated by finite element method, utilizing real geometries and material parameters obtained from robocast silicon carbide samples. Six types of robocast geometries are studied, showing different material symmetries given by the arrangement of the ceramic rods in the scaffold structures. Due to the macroscopic periodicity of the structures composed of fully sintered ceramic rods, the robocast scaffolds exhibit metamaterial-like elastic and acoustic properties, never observed for natural materials. The effect of the micro-architecture is shown to be crucial: while for tetragonal and orthorhombic structures, strong acoustic focusing along the directions of the rods appears even in the low-frequency limit, hexagonal structures exhibit no energy focusing up to some frequency limit given by the geometry.
\end{abstract}

DOI: 10.12693/APhysPolA.134.799

PACS/topics: 43.20.Gp, 43.35.Cg, 81.05.Je, 81.20.Ev

\section{Introduction}

Additive manufacturing methods are capable of producing novel advanced materials by layer-by-layer deposition, following a printing route defined by computeraided design (CAD) [1-4]. Due to their artificial, highly symmetric structure, the fabricated scaffolds exhibit unique acoustic properties, which are not observed in natural materials, e.g. acoustic frequency bandgaps [59] and strong acoustic energy focusing [10]. Moreover, such materials can be utilized as light-weight structural materials [11-13] or in bone tissue engineering $[1,14]$.

Robocasting is an additive manufacturing technique in which the microscaffolds consisting of thin rods are fabricated from a powder slurry $[15,16]$. In this paper, robocast silicon carbide $(\mathrm{SiC})$ samples were used as templates for the finite element method (FEM) modeling of elastic and acoustic properties of such microarchitectured scaffolds. It is shown that several types of ceramic scaffolds exhibit anisotropic elastic and acoustic properties leading to strong acoustic energy focusing along the principal symmetry axes of the robocast samples.

\section{Materials and methods}

A detailed description of $\mathrm{SiC}$ scaffolds fabrication by the robocasting method can be found in $[15,16]$. In brief, a colloidal gel ink, based on $\mathrm{SiC}$ powder, is extruded

*corresponding author; e-mail: martin.koller@fjfi.cvut.cz

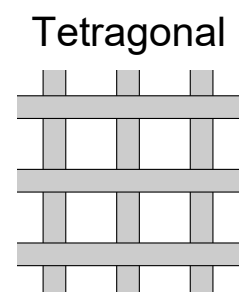

(a) LS $1 \mathrm{~mm}$

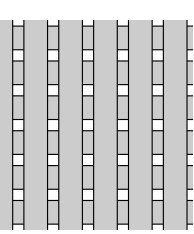

(b) SS

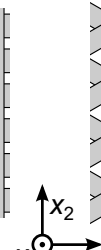

$x_{3}^{\odot} \overrightarrow{x_{1}}$

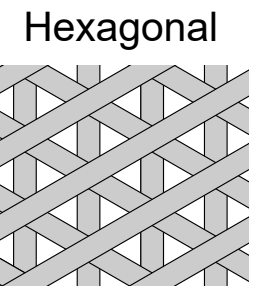

(c) $\mathrm{LH}$

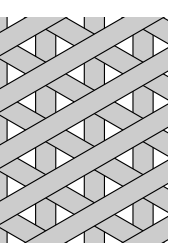

(d) $\mathrm{SH}$

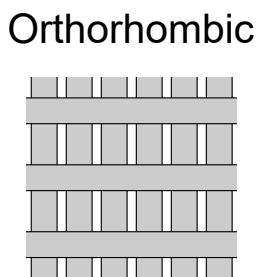

(e) $\mathrm{RO}$

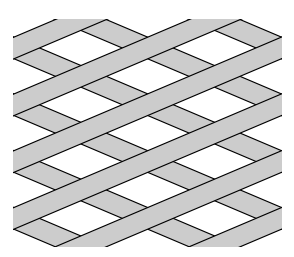

(f) $\mathrm{AO}$
Fig. 1. Geometry of microarchitectures of robocast silicon carbide scaffolds used for the FEM calculations. Tetragonal structures are denoted as (a) LS and (b) SS, meaning large square and small square, respectively. Hexagonal structures are denoted as (c) LH and (d) SH, meaning large hexagonal and small hexagonal, respectively. Orthorhombic structures are denoted as (e) RO and (f) AO, meaning rectangular orthorhombic and angular orthorhombic, respectively.

through a nozzle in an oil bath, following a layer-bylayer printing route defined by CAD. After drying and burning-out of the organic additives, the scaffolds are consolidated by presureless spark plasma sintering.

Six types of robocast $\mathrm{SiC}$ scaffolds, as shown in Fig. 1, are used as templates for FEM. Within each layer of every scaffold, the rods with diameter $d$ are mutually 
Geometric characteristics of robocast $\mathrm{SiC}$ scaffolds: angles between rods in neighbouring layers, in-plane spacings between rods $l$, rod diameters $d$, out-of-plane spacings $h$ between rods in neighboring layers; and effective densities $\rho$ computed by FEM

\begin{tabular}{c|c|c|c|c|c|c}
\hline \hline Symmetry & Sample & Angle & $l[\mu \mathrm{m}]$ & $d[\mu \mathrm{m}]$ & $h[\mu \mathrm{m}]$ & $\rho\left[\mathrm{g} \mathrm{cm}^{-3}\right]$ \\
\hline \multirow{2}{*}{ tetragonal } & LS & $90^{\circ}$ & 650 & 210 & 137.5 & 1.212 \\
& $\mathrm{SS}$ & $90^{\circ}$ & 308 & 219 & 130.5 & 2.555 \\
\hline \multirow{2}{*}{ orthorhombic } & $\mathrm{RO}$ & $90^{\circ}$ & $591 / 309.3$ & 238 & 148.7 & 2.165 \\
& $\mathrm{AO}$ & $46^{\circ}$ & 462 & 186 & 125 & 1.458 \\
\hline \multirow{2}{*}{ hexagonal } & $\mathrm{LH}$ & $60^{\circ}$ & 562 & 210 & 182 & 1.117 \\
& $\mathrm{SH}$ & $60^{\circ}$ & 430 & 188 & 150 & 1.391
\end{tabular}

parallel with in-plane spacing $l$, and the arrangement of neighboring layers is also modified, resulting in three types of material symmetries. For the two samples with tetragonal symmetry, an angle between rods of neighboring layers is equal to $90^{\circ}$; and for the two samples with hexagonal symmetry, an angle between rods of neighboring layers is equal to $60^{\circ}$. One of the samples exhibiting orthorhombic symmetry has two different values of inplane spacing of the rods with an angle between rods of neighboring layers equal to $90^{\circ}$, Fig. 1 e, and the direction of the rods in the other sample alternates positively/negatively by $46^{\circ}$ with the equal in-plane spacing and the periodicity of two layers, Fig. 1f. Out-of-plane spacings of the rods $h$ between the neighboring layers are smaller than the rod diameters $d$ for all scaffolds, which indicates significant overlapping of the $\mathrm{SiC}$ rods at their intersections. The geometric parameters $l, d, h$ of the sintered scaffolds are summarized in Table I.

Elastic coefficients $C_{i j k l}$ of all six types of robocast scaffolds were obtained by FEM, utilizing COMSOL Multiphysics software, as described in $[10,17]$. Computational unit cells were designed from the real geometry parameters shown in Table I, to represent periodic structures of the robocast scaffolds. Elastic responses were then calculated by utilizing pure tensile or pure shear straining modes along the principal directions of the unit cells. The density of $3.34 \mathrm{~g} \mathrm{~cm}^{-3}$, Young's modulus of $305 \mathrm{GPa}$, and Poisson's ratio of 0.19 of $\mathrm{SiC}[6,10]$ were set as inputs for the calculations.

From the elastic coefficients $C_{i j k l}$, phase velocity $v_{\phi}$ and group velocity vector $\boldsymbol{v}_{\boldsymbol{G}}$ are calculated. Using the Christoffel equation

$$
\left(C_{i j k l} n_{j} n_{l}-\rho v_{\phi}^{2} \delta_{i k}\right) U_{k}=0,
$$

phase velocities $v_{\phi}$ are obtained in a given direction of propagation $\boldsymbol{n}$, where $\rho$ is the effective mass density, $\delta_{i k}$ is Kronecker's delta, and $U_{k}$ is polarization vector. For each direction of propagation $\boldsymbol{n}$, three phase velocities $v_{\phi}$ with three different polarization vectors are obtained. Then, the group velocity vector is calculated as

$$
v_{G i}=C_{i j k l} n_{k} U_{j} U_{l} /\left(\rho v_{\phi}\right) .
$$

\section{Results and discussion}

In Fig. 2 and Fig. 3, the polar plots of phase and group velocities in $x_{1} x_{2}$ plane are shown for the tetragonal samples. The outer velocity curves (red in color version) represent quasi-longitudinal waves $(q L)$ and the other two curves (blue in color version) represent quasitransverse waves $(q T)$ with horizontal and vertical polarizations. The group velocities are plotted as discrete points, in order to clearly demonstrate acoustic energy focusing.

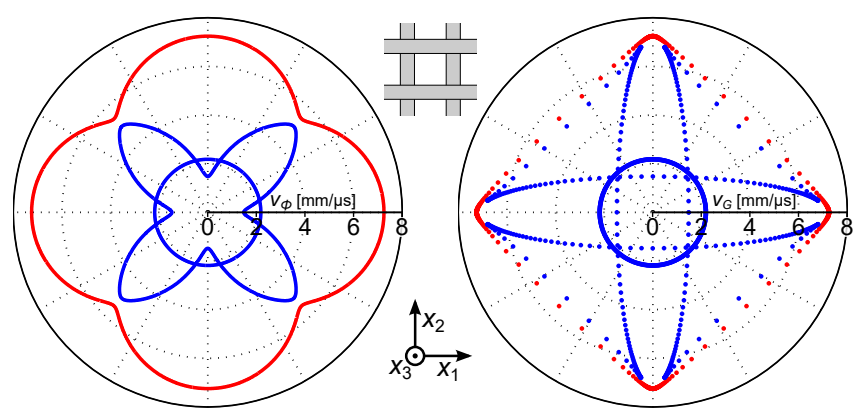

Fig. 2. Phase velocity $v_{\phi}$ and group velocity $v_{G}$ in the $x_{1} x_{2}$ plane for the LS sample. Number of group velocity data points: $3 \times 480$.

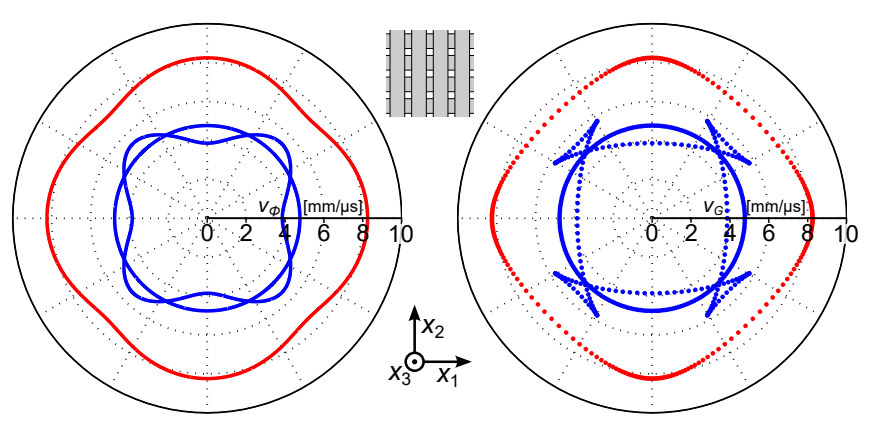

Fig. 3. Phase velocity $v_{\phi}$ and group velocity $v_{G}$ in the $x_{1} x_{2}$ plane for the SS sample. Number of group velocity data points: $3 \times 240$.

For the LS sample, phase velocity values of $q L$ and $q T$ waves are very close to each other in the $45^{\circ}$ direction, as a result of relatively low values of $c_{12}$ and $c_{13}$ coefficients due to the weak interlinking between rods. Very similar results were obtained, when the elastic coefficients of another robocast tetragonal sample [10] were determined by measuring resonant spectra of free elastic vibrations by resonant ultrasound spectroscopy method. This sample had similar average rod diameter $d$, slighty higher 
out-of-plane spacings of the rods $h$, and slightly smaller in-plane spacings of the rods $l$ than the LS sample. For both the LS sample and the sample described in [10], the $q L$ and $q T$ phase velocity values form ring-like patterns in the $x_{1} x_{2}$ symmetry plane, suggesting that the acoustic waves can be expected to propagate nearly independently in the individual rods.

For the SS sample, the phase velocities values of $q L$ and $q T$ waves significantly differ even in the $45^{\circ}$ direction, as seen in Fig. 3, suggesting that there is a much higher interlinking in this sample. As the both rod diameters $d$ and out-of-plane spacings of the rods $h$ are almost identical for the LS and SS samples, the stronger energy focusing for the LS sample has to be attributed to the much higher in-plane spacing between the rods $l$.

The acoustic energy focusing along the principal axes of the rods, $x_{1}$ and $x_{2}$, is also seen from the group velocity plots, where the vast majority of group velocity vectors of the $q L$ waves lie along these principal axes. Only when the direction of propagation $\boldsymbol{n}$ is very close to the $45^{\circ}$ angle for the LS sample, the acoustic energy propagates between the rectangular rods, but with much smaller group velocity. The focusing of $q L$ waves is more pronounced for the LS sample, as compared to the SS sample, which could be demonstrated from the density of group velocity points.

The strong acoustic energy focusing is also evident for the $q T$ waves of the LS sample. Similarly to the longitudinal waves, the directions of group velocity vector of one of the $q T$ waves lie along the principal axes for the majority of the directions of propagation $\boldsymbol{n}$. The group velocities of the second $q T$ wave are equidistantly distributed with a constant value, indicating an independent projection to $x_{1} x_{2}$ symmetry plane, which suggests that the polarization vector of this $q T$ wave lies along the $x_{3}$ principal axis; in Ref. [10], such waves are designated as pure transverse (PT). Likewise for the $q L$ waves, the energy focusing of the $q T$ waves is more pronounced for LS sample, when compared to SS sample.

Similar velocity curves with the $q L$ energy focusing along the principal axes are observed for the RO sample, in Fig. 4. Since this sample has two different in-plane spacings of the rods, the group velocities along the principal axes differ, where the higher group velocity value corresponds to the direction with a closer arrangement of parallel rods in the $x_{1}$ axis. Moreover, the pattern of the second $q T$ wave is not circle-like for this sample, unlike for the tetragonal samples, indicating that this wave is not pure transverse, but rather quasi-transverse as a result of the lower symmetry class of the orthorhombic sample.

For the AO sample, Fig. 5, distinguished energy focusing in the directions between the smaller angle between rods, which the axis $x_{1}$ intersect in half, is observed for both $q L$ and $q T$ waves.

For the hexagonal samples, Figs. 6 and 7, no energy focusing in the $x_{1} x_{2}$ plane is observed, since the group velocity vectors are uniformly distributed in this

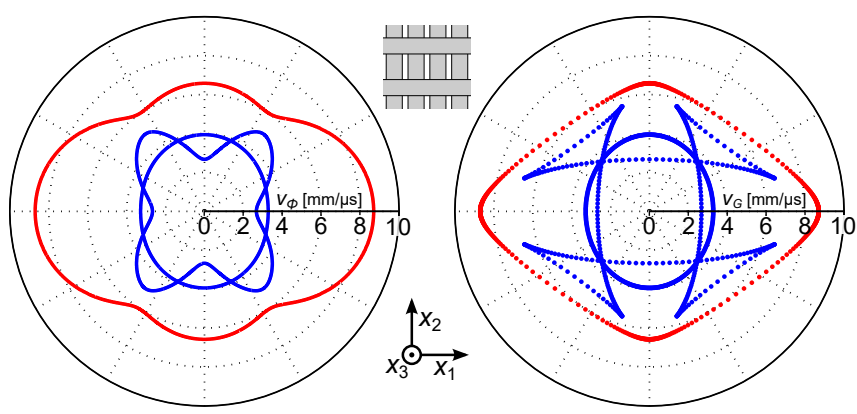

Fig. 4. Phase velocity $v_{\phi}$ and group velocity $v_{G}$ in the $x_{1} x_{2}$ plane for the RO sample. Number of group velocity data points: $3 \times 480$.

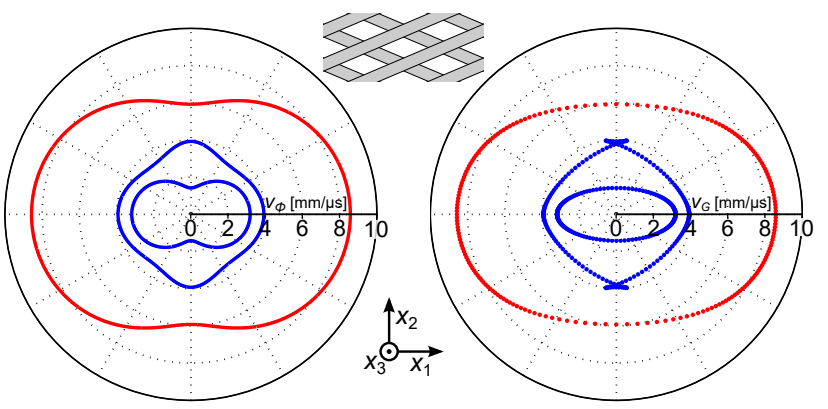

Fig. 5. Phase velocity $v_{\phi}$ and group velocity $v_{G}$ in the $x_{1} x_{2}$ plane for the AO sample. Number of group velocity data points: $3 \times 200$.

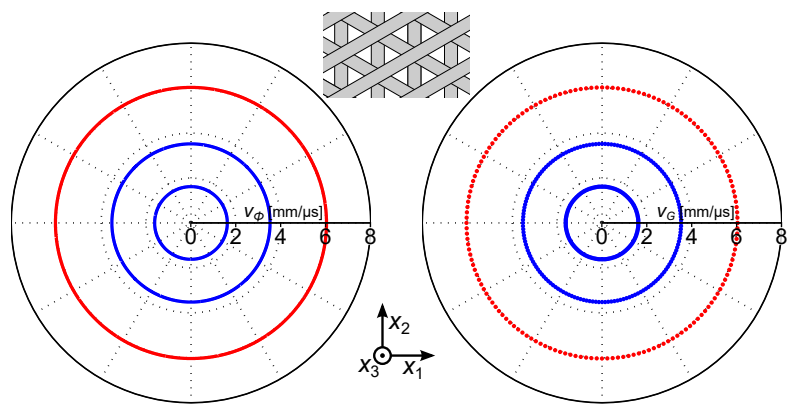

Fig. 6. Phase velocity $v_{\phi}$ and group velocity $v_{G}$ in the $x_{1} x_{2}$ plane for the LH sample. Number of group velocity data points: $3 \times 160$.

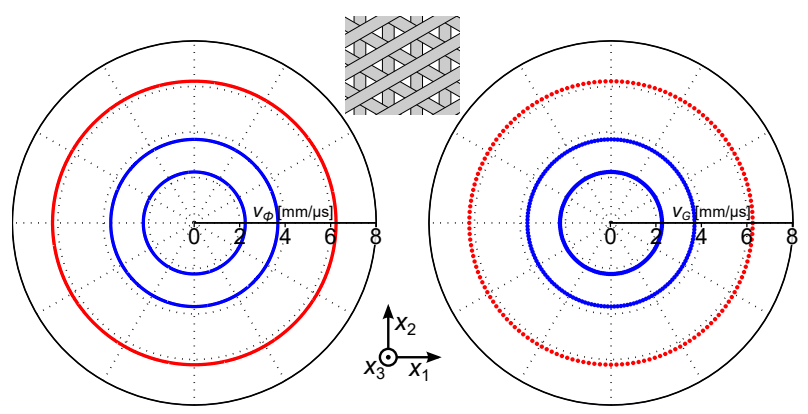

Fig. 7. Phase velocity $v_{\phi}$ and group velocity $v_{G}$ in the $x_{1} x_{2}$ plane for the SH sample. Number of group velocity data points: $3 \times 160$. 
plane with constant magnitudes. This is an expected result, because hexagonal materials have the same macroscopic symmetry, with 5 independent elastic coefficients, as transverse isotropic materials, in which the material properties are independent of direction in the isotropic plane of $x_{1} x_{2}$ [18].

Nevertheless, some acoustic energy focusing can be still expected due to the macroscopic heterogeneous nature of the LH sample, if the wavelengths are comparable to the spacing of the rods. Therefore, the FEM was also utilized for calculating properties of planar waves, where the displacement field $\boldsymbol{u}$ can be assumed in the form

$$
\boldsymbol{u}(\boldsymbol{x}, t)=\boldsymbol{U}(\boldsymbol{x}, \boldsymbol{k}) \exp [\mathrm{i}(\boldsymbol{k} \cdot \boldsymbol{x}-2 \pi f t)] .
$$

$\boldsymbol{k}$ is wave vector, $f$ is frequency, and $\boldsymbol{U}(\boldsymbol{x}, \boldsymbol{k})$ is the Bloch waveform having the same spatial periodicity as the scaffold. Due to the 12 symmetry planes of the LH sample, Fig. 1c, the directions of wave propagation $\boldsymbol{k}$ were chosen as $\alpha=0^{\circ}, 5^{\circ}, 10^{\circ}, 15^{\circ}, 20^{\circ}, 25^{\circ}, 30^{\circ}$ angles with respect to the $x_{1}$ axis. For each direction, the set of wave vector magnitudes $|\boldsymbol{k}|$, ranging from 0 to $\pi /(l \cos \alpha)$, was taken. Using Eq. (3), the frequencies $f$ corresponding to particular $\boldsymbol{k}$ were calculated on the basic computational unit cells with the complex phase-shift periodic boundary conditions. The phase shift was given by the phase factor $\exp (\mathrm{i}(\boldsymbol{k} \cdot \boldsymbol{x}))$ from Eq. (3).

In Fig. 8, frequency dependence on the wave vector magnitudes is shown for all three types of acoustic waves (one $q L$ wave and two $q T$ waves). As seen in this figure, if the $\boldsymbol{k}$ magnitudes are much smaller than $\pi / l$, e.g. if the wavelength is significantly longer than the in-plane spacing of the rods $l$, all directions of propagation lead to very similar frequency values, and thus the hexagonal structure seems to be isotropic in the $x_{1} x_{2}$ plane in the low-frequency limit. With the increasing $\boldsymbol{k}$ magnitudes, the frequency curves become dependent on direction of wave propagation, where the highest frequency at given wave vector and thus the largest phase velocity value corresponds to the propagation along the principal axes of the $\mathrm{SiC}$ rods. Therefore, when the wavelengths become comparable to the in-plane spacing of the rods, acoustic energy is more focused along the ceramic rods. The energy focusing is also demonstrated in the polar plots of phase and group velocities at the frequency of $1 \mathrm{MHz}$, as shown in Fig. 9.

\section{Conclusion}

Acoustic wave propagation in robocast $\mathrm{SiC}$ scaffolds was calculated by FEM, utilizing real scaffold geometries and $\mathrm{SiC}$ material properties. The geometry of the tetragonal and orthorhombic scaffolds is shown to be crucial for acoustic energy focusing along the principal axes of $\mathrm{SiC}$ rods. The strongest acoustic focusing was observed for the tetragonal sample with the highest value of in-plane spacings between rods, suggesting that the acoustic energy is more focused in the least dense structures. For the hexagonal scaffolds, the effect of the geometry is significant only when the wavelengths are comparable to the

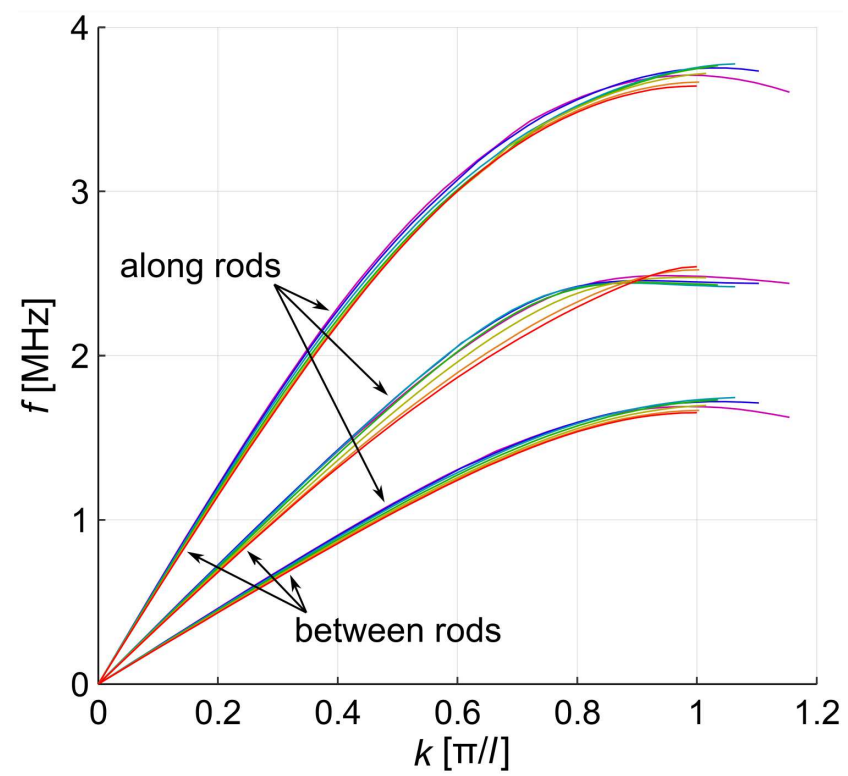

Fig. 8. Frequency $f$ vs. the magnitudes $k$ of wave vector at several directions of wave propagation $\boldsymbol{n}$. The top group of curves represents quasi-londitudinal waves and the other two groups of curves represent quasitransverse waves.

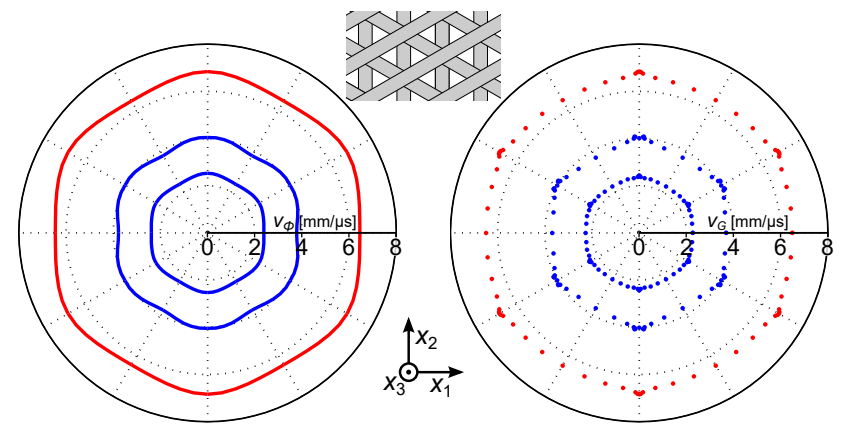

Fig. 9. Phase velocity $v_{\phi}$ and group velocity $v_{G}$ in the $x_{1} x_{2}$ plane for the LH sample, as calculated from the acoustic wave propagation at the frequency of $1 \mathrm{MHz}$.

spacings between rods, e.g. when the wave frequency is sufficiently high. In the low-frequency limit, the hexagonal scaffolds are isotropic in the hexagonal plane due to their higher degree of macroscopic material symmetry.

The presented result prove that the acoustic properties of robocast materials can be effectively tailored by designing the geometry of the scaffolds. These materials are therefore prospective candidates for applications as smart materials for focusing and redirecting of acoustic energy.

\section{Acknowledgments}

This work was supported by the Czech Science Foundation (Grant No. GA17-01618S), and by Spanish project MAT2015-67437-R (MINECO, FEDER, UE). 


\section{References}

[1] Q. Fu, E. Saiz, M.N. Rahaman, A.P. Tomsia, Mater. Sci. Eng. C Mater. Biol. Appl. 31, 1245 (2011).

[2] B.G. Compton, J.A. Lewis, Adv. Mater. 26, 5930 (2014).

[3] J.R. Raney, J.A. Lewis, MRS Bull. 40, 943 (2015).

[4] M.K. Thompson, G. Moroni, T. Vaneker, G. Fadel, R.I. Campbell, I. Gibson, A. Bernard, J. Schulz, P. Graf, B. Ahuja, F. Martina, CIRP Ann. Manuf. Technol. 65, 737 (2016).

[5] F. Lucklum, M.J. Vellekoop, Proced. Eng. 120, 1095 (2015).

[6] A. Kruisová, M. Ševcík, H. Seiner, P. Sedlák, B. Román-Manso, P. Miranzo, M. Belmonte, M. Landa, Ultrasonics 82, 91 (2018).

[7] T. Delpero, S. Schoenwald, A. Zemp, A. Bergamini, J. Sound Vibrat. 363, 156 (2016).

[8] K.H. Matlack, A. Bauhofer, S. Krödel, A. Palermo, C. Daraio, Proc. Natl. Acad. Sci. USA 113, 8386 (2016).

[9] H. Seiner, P. Sedlák, M. Ševcík, A. Kruisová, M. Landa, B. Román-Manso, P. Miranzo, M. Belmonte, in: Proc. VIII ECCOMAS Thematic Conf. on Smart Structures and Materials SMART 2017, Eds. A. Güemes, A. Benjeddou, J. Rodellar, J. Leng, CIMNE, Barcelona 2017, p. 1702.
[10] A. Kruisová, H. Seiner, P. Sedlák, M. Landa, B. Román-Manso, P. Miranzo, M. Belmonte, Appl. Phys. Lett. 105, 211904 (2014).

[11] R. Huang, M. Riddle, D. Graziano, J. Warren, S. Das, S. Nimbalkar, J. Cresko, E. Masanet, J. Clean. Prod. 135, 1559 (2016).

[12] Y. Tang, Y.F. Zhao, Rapid Prototyp. J. 22, 569 (2016).

[13] C.B. Williams, J.K. Cochran, D.W. Rosen, Int. J. Adv. Manuf. Technol. 53, 231 (2011).

[14] P. Miranda, E. Saiz, K. Gryn, A.P. Tomsia, Acta Biomater. 2, 457 (2006)

[15] K. Cai, B. Román-Manso, J.E. Smay, J. Zhou, M.I. Osendi, M. Belmonte, P. Miranzo, J. Am. Ceram. Soc. 95, 2660 (2012).

[16] B. Román-Manso, Á. de Pablos, M. Belmonte, M.I. Osendi, P. Miranzo, Bol. Soc. Esp. Ceram. Vidr. 53, 93 (2014).

[17] A. Kruisová, H. Seiner, P. Sedlák, M. Landa, B. Román-Manso, P. Miranzo, M. Belmonte, Appl. Mech. Mater. 821, 364 (2016).

[18] H. Seiner, C. Ramirez, M. Koller, P. Sedlák, M. Landa, P. Miranzo, M. Belmonte, M.I. Osendi, Mater. Des. 87, 675 (2015). 